\title{
Antinutrient content, vitamin constituents and antioxidant properties in some value-added Nigerian traditional snacks
}

\author{
O. Aletor ${ }^{1}$, G. Oboh $^{2}$ \& S. F. Ojo ${ }^{1}$ \\ ${ }^{1}$ Department of Chemistry, \\ The Federal University of Technology, Nigeria \\ ${ }^{2}$ Department of Biochemistry, \\ The Federal University of Technology, Nigeria
}

\begin{abstract}
Popularly consumed traditional snacks kulikuli (groundnut-based) and kokoro (from whole maize) consumed mainly in the Northern and South-western parts of Nigeria respectively, were enriched with some well known nutraceuticals: pepper (Capsicum annuum); ginger (Zingiber offinale); sweet basil (Ocimum gratisimum); orange peel (Citrus sinensis); cocoa powder (Theobroma cacao); soya beans (Glycine max) and roselle (Hibiscus sabdariffa) in the ratio 1 or $2 \%$. The enriched and non-enriched (control) snacks were analysed and compared with respect to their sensory qualities (to ascertain consumer's acceptability) using a 7-point hedonic scale, antinutrient content, vitamins $\mathrm{A}$ and $\mathrm{C}$ and antioxidant properties. The sensory attributes of all the enriched snacks were accepted as good, very good and extremely good except with the addition of ginger which had average acceptability. Results on the anti-nutrients showed that relative to the controls, $2 \%$ addition of cocoa powder or pepper to kokoro and kulikuli, significantly increased phytic acid content ( $\mathrm{p} \leq 0.05 ; 6.59$ vs. 8.24mg/g phytic acid and 6.47 vs. $7.55 \mathrm{mg} / \mathrm{g}$ phytic acid) respectively. The study showed that nutraceutical enrichment significantly $(\mathrm{p} \leq 0.05)$ increased vitamins $A$ in kokoro and kulikuli at $1 \%$ addition in the range 2290.35-2780.35units/g; 2914.35-3582.52units/g, respectively when compared with the controls. Kulikuli enrichment at 2\% level increased vit C content from 1.73 to $2.65 \mathrm{mg} / \mathrm{g}$ as compared with kokoro (4.53 to $6.07 \mathrm{mg} / \mathrm{g}$ ). The total phenolic content, reducing ability and free-radical scavenging ability were higher in the enriched snacks than the control. The free phenolic extracts from enriched snacks had a
\end{abstract}


significantly higher $(\mathrm{p} \leq 0.05)$ antioxidant activity than the non-enriched products. Results suggest that value addition to these traditional snacks especially with ginger, sweet basil and roselle confers on them higher functionality by increasing their vitamins and antioxidant activity in addition to their traditional role as snacks.

Keywords: value-added traditional snacks, sensory attributes, antinutrients, antioxidant properties.

\section{Introduction}

The micro-nutrients deficit in foods arise not only as a consequence of increasing population without a corresponding increase in food production, but also due to the lack of economic access to the relatively more expensive conventional an important nutrient-enriched food items needed for healthy and productive living. The development of cheap and value added traditional snacks and food products may be strategic approach towards the management of micro-nutrient deficiency and related diseases. These types of products are commonly known as functional foods, defined as foods similar in appearance to conventional foods that are consumed as part of the normal diet and have demonstrated physiological benefits and/or reduced the risk of chronic diseases beyond basic nutritional factors (Zhang et al. [1]).

Antioxidants are powerful free radical scavengers in the body, while free radicals are highly reactive chemical substances such as superoxide, hydroxyl radical, singlet oxygen, etc. (Zhang et al. [1]) that travel round the body and may oxidize nuclei acid, proteins, lipids or DNA and can initiate degenerative diseases. Human body is equipped with an antioxidant defence system that deactivates these highly reactive free radicals. Antioxidants enzymes (made in the body) and antioxidant nutrients (found in foods) soak up all the excess energy [2]. Current thinking among the natural health community indicates that supplements of vitamins and other plant-based chemical (phytochemicals) are important to ensure that we do not suffer from heart disease, cancer or other chronic diseases [3].

Plant foods, including fruits, vegetables and grains are good for us. Potter [3] confirms that some of these foods do, as part of an overall health diet, have the potential to delay the onset of many age related diseases and this appear to be due to high levels of antioxidants and other phytochemicals [2]. In recent years, cereals and its ingredients are accepted as functional foods and nutraceuticals because they are rich in dietary fibre, proteins, energy, minerals, vitamins and antioxidants required for human health [2]. Therefore, this study seeks to produce traditional snacks and cakes (kulikuli and kokoro) enriched with established nutraceuticals such as pepper, ginger, sweet basil, orange peel, cocoa powder, soya beans and roselle and to investigate the effect of value addition on the antinutrients, vitamins and antioxidant properties. 


\section{Materials and methods}

\subsection{Sample collection and preparation}

Maize (corn) grains and groundnut were purchased from a local market in Akure, Nigeria. They were authenticated in the Department of Crop, Soil and Pest Management of The Federal University of Technology Akure, Nigeria. Maize grains and groundnut were cleaned, air-dried, and ground into a powdery form.

\subsection{Production of value-added kokoro}

The whole maize grains were cleaned and milled. The milled samples were later mixed/enriched with grounded forms of some established nutraceuticals (pepper, ginger, sweet basil, orange peel, cocoa powder, soyabeans and roselle extracts) at 1 or $2 \%$ ratio and boiled with continuous stirring. The mixture is allowed to cool and then kneaded for 5 minutes, roll into shape and fried for 3 minutes, cooled and packaged into a polyethylene bag.

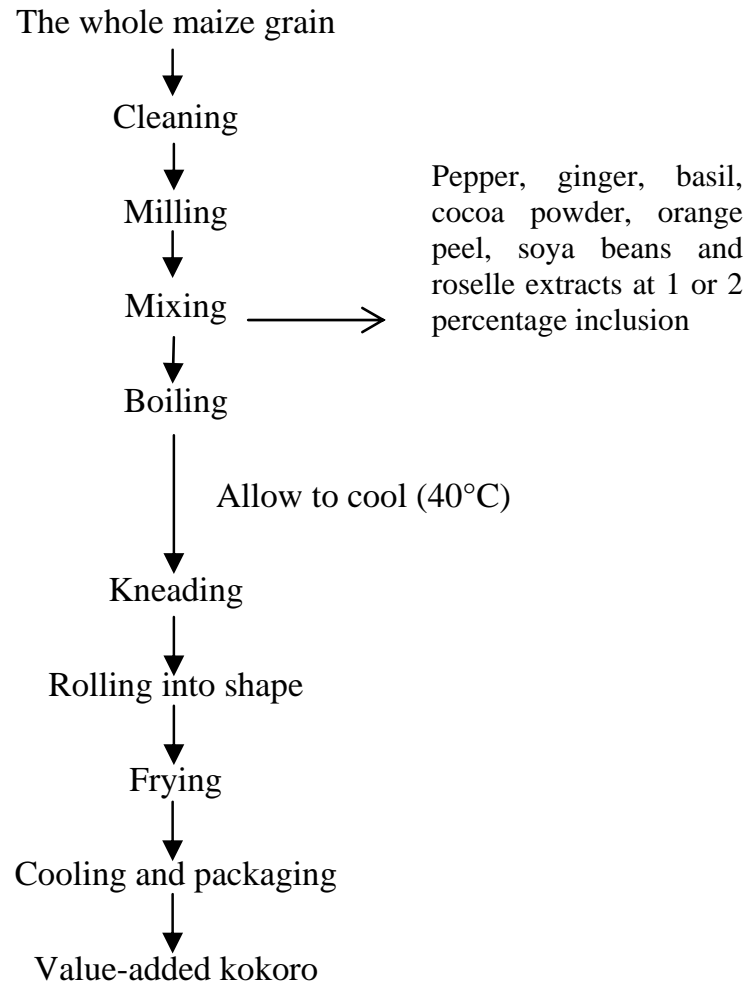

Figure 1: $\quad$ Production of value-added kokoro. 


\subsection{Production of value-added kulikuli}

Groundnut was first shelled, cleaned, roasted and dehulled and grounded and pressed to remove as much oil as possible. The resultant cake was then seasoned by addition of some established nutraceuticals (pepper, ginger, sweet basil, orange peel, cocoa powder, soyabeans and roselle extracts) and deep fat frying. The flow chart is shown in Fig. 2.

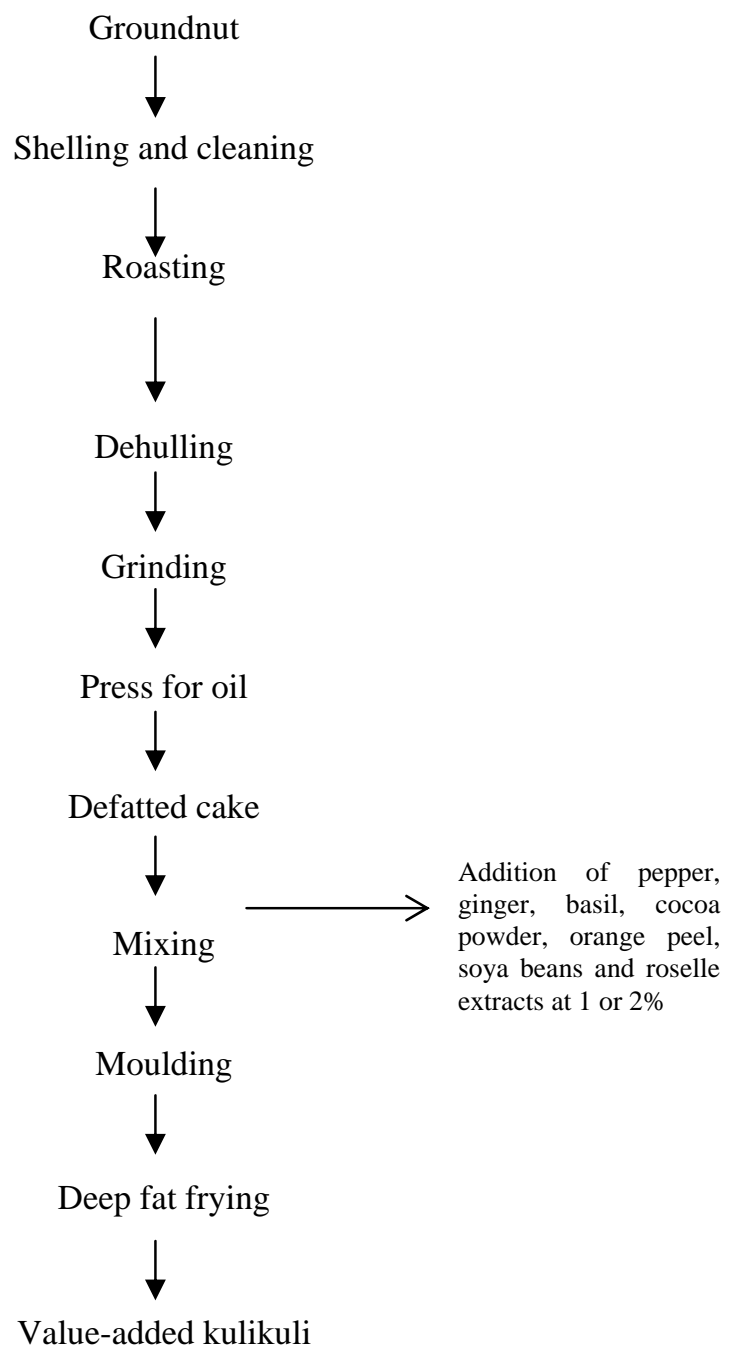

Figure 2: $\quad$ Production of value-added kulikuli. 


\subsection{Methods}

\subsubsection{Sensory analysis}

The organoleptic properties of the snacks and cakes carried out using the method of [3]. The product were assessed (aroma, taste, texture, color and general acceptability) on a 7-point Hedonic scale $(7=$ excellent, $6=$ very good, $5=$ good, 4 = average, 3 = fair, 2 = poor, 1 = very poor) as described by [3] and the attribute mean score was calculated.

\subsubsection{Determination of antinutrient composition}

The phytate content was determined by the method of Wheeler and Ferrel [4], based on the ability of standard ferric chloride to precipitate phytate in dilute $\mathrm{HCl}$ of the snacks and cakes. Tannin content was determined using the method of Makkar et al. [5].

\subsubsection{Determination of vitamin a and vitamin $c$}

$\beta$-carolene (vit. A) was determined using acetone-petroleum ether $\left(40-60^{\circ} \mathrm{C}\right)$ in the ratio of $1: 3$ as a solvent as described by [6]. The quantification was done by the HPLC (model water S:600) multi-solvent delivery system and a 996 photodiode array detector PDA at 452nm. The attached computer with millennium PDA software enables the use of millennium chromatography manager to acquire and process spectral and chromatographic data. Analytical column YMC-Pack C30S-5mm, 250 X 4.6mm i.d. and mobile phase methanol $56 \mathrm{~g} \mathrm{~kg}^{-1}$ tertiary butylmethyl ether $40 \mathrm{~g} \mathrm{~kg}^{-1}$ and water $4 \mathrm{~g} \mathrm{~kg}$ was used. Ascorbic acid (Vit. C) was determined by titration against 2, 6-dichlorophenol indophenols as described by [7].

\subsubsection{Determination of anti-oxidant properties}

Aqueous extract of the control and the value added snacks were prepared in hot water; briefly about $1 \mathrm{~g}$ each of the samples was soaked in $100 \mathrm{ml}$ hot water for 5 min. Thereafter, the mixtures were centrifuged at 2,000 rpm for $10 \mathrm{~min}$. The supernatant was used for the determination of total phenol and antioxidant activity

\subsubsection{Total phenol determination}

The total phenol content was determined by adding $0.5 \mathrm{ml}$ extract to $2.5 \mathrm{ml} 10 \%$ Folin-Ciocalteu's reagent (v/v) and $2.0 \mathrm{ml} \mathrm{7.5 \%} \mathrm{NaCO}_{3}$ added. The mixture was incubated at $45^{\circ} \mathrm{C}$ for $40 \mathrm{~min}$. and the absorbance was measured at $765 \mathrm{~nm}$ in a spectrophotometer using gallic acid as a standard as described by Singleton et al. [7].

\subsubsection{Free radical scavenging ability}

The free radical scavenging ability of the vegetables against 1, 1-diphenyl-2picrylhydrazyl (DPPH) free radical was also evaluated as described by Ursini et al. [8]. Briefly, a 1-mL aliquot $0.05 \mathrm{~g}$ of the extract was dissolved in $20 \mathrm{~mL}$ of methanol was mixed with $1 \mathrm{~mL}$ of $0.4 \mathrm{~m}$ methanolic solution containing $\mathrm{DPPH}$ 
radicals, and the mixture was left in the dark for 30 minutes before the absorbance at $516 \mathrm{~nm}$ was measured.

\subsubsection{Analysis of data}

One way analysis of variance (ANOVA) and the least significance difference (LSD) were carried out [9]. Significance was accepted at $\mathrm{P} \leq 0.05$

\section{Result and discussion}

The results of the sensory evaluation of the control and value added kokoro and kulikuli are shown in Table 1. All the value-added traditional snacks had a lower overall acceptability when compared to the control. However, among the value added produced snacks (kokoro and kulikuli) products with pepper, and sweet basil had the highest general acceptability (taste, texture, colour, aroma and texture) and closely followed with soya beans, orange peel, cocoa powder, ginger while the $H$. sabdariffa addition had the least acceptability. The levels of antinutrients (phytate and Tanin) in the control and value-added kulikuli and kokoro are shown in Table $2 \mathrm{a}$ and Table $2 \mathrm{~b}$ respectively. The result showed that relative to the control $6.47 \mathrm{mg} / \mathrm{g}$ phytin at $1 \%$ addition of nutraceutical enrichment in kokoro ranged 6.65 (C. sinesis) to $7.02 \mathrm{mg} / \mathrm{g}$ (C. annum). At 2\% addition ranged 7.04 (T. cacao) to $7.55 \mathrm{mg} / \mathrm{g}$ (C. annuum) and the highest values at 1 and 2\% change are $8.50 \%$ (pepper) and 16.69\% (pepper) respectively.

Result on kulikuli (Table 2b) on phytin showed that there was significant difference $\mathrm{P}<0.05$ at 1 or $2 \%$ addition ranged $16.62 \mathrm{mg} / \mathrm{g}$ (Z. officinale) to $7.42 \mathrm{mg} / \mathrm{g}$ (T. cacao) and $6.95 \mathrm{mg} / \mathrm{g}$ (G. max) to $8.24 \mathrm{mg} / \mathrm{g}$ (T. cacao) respectively. The highest 1 or $2 \%$ change in kulikuli was $21.33 \%$ (pepper) $33.88 \%$ (pepper) and kokoro had $12.90 \%$ (T. cacao and O. gratisinum) $24.74 \%$ (T. cacao) respectively. Tannin content in kulikuli and kokoro Tables 2a and 2b showed similar value on the controls 0.84 and $0.90 \mathrm{~g} / 100 \mathrm{~g}$ respectively with no significant increase $\mathrm{P}>0.05$ at 1 or $2 \%$ addition while 1 or $2 \%$ change revealed a significantly $\mathrm{P}<0.05$ increased in tannic acid in kulikuli (29.6\%), kokoro (50.0\%) at $2 \%$ addition of O. gratisinum and H. sabdariffa respectively.

The study showed that nutraceutical enrichment increased the antinutrient content of these African traditional snacks. However, phytic acid though considered as an antinutritional factor is a common storage of phosphorus in plant foods [2]. The complexing of phytic acid with nutritionally essential minerals is suggested as being responsible for the antinutritional activity. The phosphorus in phytic acid is not nutritionally available to monogastric animals. Phytic acid interferes with $\mathrm{Ca}, \mathrm{Fe}, \mathrm{Mg}$ and $\mathrm{Zn}$ absorption because of its ability to chelate divalent cationic minerals. As shown in the tables 2a and 2b (kulikuli and kokoro) the highest value at $2 \%$ percentage change was kulikuli $(16.69 \%$ pepper). This value is far below the phytate content of S. sparganophora leaf $225.6 \mathrm{mg} / 100 \mathrm{~g}$ reported by [2]. Tannins are complex polyphenolics found widely in the plant kingdom. It brings about anti-nutritional influence largely by forming complexes and thus precipitating dietary proteins and digestive enzymes. The poor palatability generally associated with high tannin diets can 

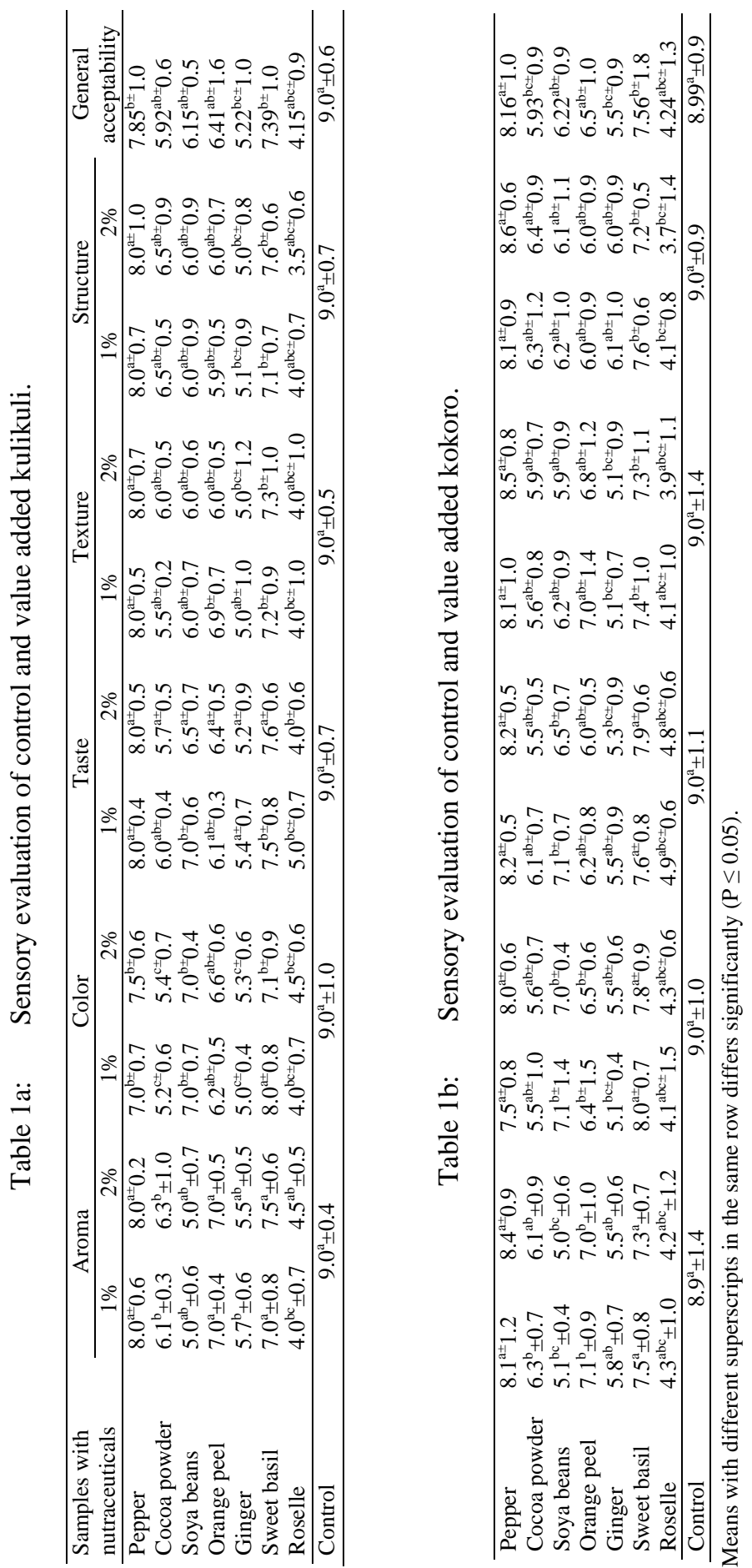
Table 2a Antinutrient composition mg/g of the control and value added kulikuli.

\begin{tabular}{llllll}
\hline \multicolumn{1}{c}{$\begin{array}{c}\text { Source of } \\
\text { nutraceuticals }\end{array}$} & Control & $1 \%$ addition & $\begin{array}{c}\text { Percentage } \\
\text { change at } 1 \% \\
\text { addition }\end{array}$ & $\begin{array}{c}2 \% \\
\text { addition }\end{array}$ & $\begin{array}{c}\text { Percentage } \\
\text { change at } \% \\
\text { addition }\end{array}$ \\
\hline $\begin{array}{l}\text { Phytin content } \\
\text { Pepper }\end{array}$ & 6.47 & $7.02^{\mathrm{a} \pm} 0.7$ & $8.50^{\mathrm{a}} \pm 0.7$ & $7.55^{\mathrm{a}} \pm 1.7$ & $16.69 \pm 1.7$ \\
Cocoa powder & 6.47 & $6.81^{\mathrm{a}} \pm 0.1$ & $5.26^{\mathrm{b}} \pm 0.1$ & $7.04^{\mathrm{a}} \pm 0.9$ & $8.81 \pm 0.9$ \\
Soya beans & 6.47 & $6.92^{\mathrm{a}} \pm 1.2$ & $6.96^{\mathrm{a}} \pm 1.2$ & $7.42^{\mathrm{a}} \pm 1.3$ & $14.68 \pm 1.3$ \\
Orange peel & 6.47 & $6.65^{\mathrm{a}} \pm 1.7$ & $2.78^{\mathrm{ab}} \pm 1.7$ & $7.07^{\mathrm{a}} \pm 1.4$ & $9.27 \pm 1.4$ \\
Ginger & 6.47 & $6.85^{\mathrm{a}} \pm 0.9$ & $5.87^{\mathrm{b}} \pm 0.9$ & $7.47^{\mathrm{a}} \pm 0.5$ & $15.46 \pm 0.5$ \\
Sweet basil & 6.47 & $6.88^{\mathrm{a}} \pm 0.6$ & $6.34^{\mathrm{b}} \pm 0.6$ & $7.51^{\mathrm{a}} \pm 1.1$ & $16.07 \pm 1.1$ \\
Roselle & 6.47 & $6.92^{\mathrm{a}} \pm 0.5$ & $6.96^{\mathrm{a}} \pm 0.5$ & $7.32^{\mathrm{a}} \pm 1.4$ & $13.14 \pm 1.4$ \\
\hline & & & & & \\
\hline Tannic acid & & & & & \\
Pepper & 0.84 & $0.85^{\mathrm{a}} \pm 0.9$ & $1.19^{\mathrm{abc}} \pm 0.9$ & $0.90^{\mathrm{a}} \pm 0.5$ & $7.14 \pm 0.5$ \\
Cocoa powder & 0.84 & $0.87^{\mathrm{a}} \pm 1.1$ & $3.57^{\mathrm{c}} \pm 1.1$ & $0.90^{\mathrm{a}} \pm 0.2$ & $7.14 \pm 0.2$ \\
Soya beans & 0.84 & $0.95^{\mathrm{a}} \pm 1.5$ & $13.10^{\mathrm{ab}} \pm 1.5$ & $1.00^{\mathrm{a}} \pm 0.3$ & $19.05 \pm 0.3$ \\
Orange peel & 0.84 & $0.86^{\mathrm{a}} \pm 1.4$ & $2.38^{\mathrm{c}} \pm 1.4$ & $0.89^{\mathrm{a}} \pm 0.6$ & $5.95 \pm 0.6$ \\
Ginger & 0.84 & $0.96^{\mathrm{a}} \pm 0.11$ & $14.29^{\mathrm{ab}} \pm 0.11$ & $0.98^{\mathrm{a}} \pm 0.6$ & $16.67 \pm 0.6$ \\
Sweet basil & 0.84 & $1.05^{\mathrm{a}} \pm 0.8$ & $25.0^{\mathrm{a}} \pm 0.8$ & $1.06^{\mathrm{a}} \pm 0.9$ & $26.19 \pm 0.9$ \\
Roselle & 0.84 & $0.98^{\mathrm{a}} \pm 0.8$ & $16.67^{\mathrm{b}} \pm 0.8$ & $1.01^{\mathrm{a}} \pm 1.4$ & $20.24 \pm 1.4$ \\
\hline
\end{tabular}

Values represent means of triplicate.

Table $2 \mathrm{~b}$ Antinutrient composition $\mathrm{mg} / \mathrm{g}$ of the control and value added kokoro.

\begin{tabular}{llllll}
\hline \multicolumn{1}{c}{$\begin{array}{c}\text { Source of } \\
\text { nutraceuticals }\end{array}$} & Control & $1 \%$ addition & $\begin{array}{c}\text { Percentage } \\
\text { change at } \% \\
\text { addition }\end{array}$ & $\begin{array}{c}2 \% \\
\text { addition }\end{array}$ & $\begin{array}{c}\text { Percentage } \\
\text { change at } \% \\
\text { addition }\end{array}$ \\
\hline $\begin{array}{l}\text { Phytin content } \\
\text { Pepper }\end{array}$ & 6.59 & $6.77^{\mathrm{a} \pm} 0.7$ & $2.73^{\mathrm{c}} \pm 0.7$ & $7.42^{\mathrm{b}} \pm 1.2$ & $12.59^{\mathrm{ab}} \pm 1.2$ \\
Cocoa powder & 6.59 & $7.42^{\mathrm{a}} \pm 0.3$ & $12.60^{\mathrm{a}} \pm 0.3$ & $8.24^{\mathrm{a}} \pm 0.9$ & $25.04^{\mathrm{a}} \pm 0.9$ \\
Soya beans & 6.59 & $6.69^{\mathrm{a}} \pm 1.0$ & $1.52^{\mathrm{abc}} \pm 1.0$ & $6.95^{\mathrm{b}} \pm 0.9$ & $5.46^{\mathrm{abc}} \pm 0.9$ \\
Orange peel & 6.59 & $6.94^{\mathrm{a}} \pm 0.7$ & $5.31^{\mathrm{ab}} \pm 0.7$ & $7.52^{\mathrm{a}} \pm 1.1$ & $14.11^{\mathrm{b}} \pm 1.1$ \\
Ginger & 6.59 & $6.62^{\mathrm{a}} \pm 0.8$ & $3.33^{\mathrm{c}} \pm 0.8$ & $7.12^{\mathrm{b}} \pm 1.6$ & $8.04^{\mathrm{c}} \pm 1.6$ \\
Sweet basil & 6.59 & $7.40^{\mathrm{a}} \pm 1.2$ & $12.44^{\mathrm{a}} \pm 1.2$ & $7.56^{\mathrm{a}} \pm 0.7$ & $14.72^{\mathrm{b}} \pm 0.7$ \\
Roselle & 6.59 & $7.31^{\mathrm{a}} \pm 1.4$ & $10.93^{\mathrm{ab}} \pm 1.4$ & $7.40^{\mathrm{b}} \pm 0.5$ & $12.29^{\mathrm{ab}} \pm 0.5$ \\
\hline & & & & & \\
\hline Tannic acid & & & & & \\
Pepper & 0.90 & $1.22^{\mathrm{a}} \pm 0.5$ & $35.56^{\mathrm{b}} \pm 0.5$ & $1.31^{\mathrm{a}} \pm 1.0$ & $41.00^{\mathrm{ab}} \pm 1.0$ \\
Cocoa powder & 0.90 & $1.11^{\mathrm{a}} \pm 0.3$ & $23.30^{\mathrm{c}} \pm 0.3$ & $1.30^{\mathrm{a}} \pm 0.9$ & $30.00^{\mathrm{abc}} \pm 0.9$ \\
Soya beans & 0.90 & $1.00^{\mathrm{a}} \pm 0.7$ & $11.11^{\mathrm{abc}} \pm 0.7$ & $1.22^{\mathrm{a}} \pm 0.9$ & $35.56^{\mathrm{c}} \pm 0.9$ \\
Orange peel & 0.90 & $1.01^{\mathrm{a}} \pm 0.7$ & $12.22^{\mathrm{abc}} \pm 0.7$ & $1.25^{\mathrm{a}} \pm 0.5$ & $35.00^{\mathrm{c}} \pm 0.5$ \\
Ginger & 0.90 & $1.32^{\mathrm{a}} \pm 0.5$ & $46.66^{\mathrm{a}} \pm 0.5$ & $1.32^{\mathrm{a}} \pm 0.6$ & $46.67^{\mathrm{b}} \pm 0.6$ \\
Sweet basil & 0.90 & $1.14^{\mathrm{a}} \pm 1.0$ & $26.66^{\mathrm{ab}} \pm 1.0$ & $1.19^{\mathrm{a}} \pm 0.7$ & $32.22^{\mathrm{abc}} \pm 0.7$ \\
Roselle & 0.90 & $1.21^{\mathrm{a} \pm 0.8}$ & $34.44^{\mathrm{b}} \pm 0.8$ & $1.35^{\mathrm{a}} \pm 0.9$ & $50.00^{\mathrm{a}} \pm 0.9$ \\
\hline
\end{tabular}

Values represent means of triplicate readings.

Values with the same letter along the same column are not significantly different $(\mathrm{P}>0.05)$. 
be ascribed to tannin's astringent property, which is a consequence of its ability to bind with the proteins of saliva and the mucosal membrane of the mouth during the mastication of foods [2]. Values on the control and value-added snacks compared well with the tannin content for cassava products (Oboh and Akindahunsi [10]). These products could also be considered to be safe with regard to tannin poisoning because the levels reported in this study are far below the critical value of $7.3-9.0 \mathrm{mg} / \mathrm{g}$ [11].

Nutraceutical enrichment significantly P $<0.05$ increased. Vitamin A Table 3 in kulikuli and kokoro at 1\% addition in the range 2918.98 (H. sabdariffa) 3582.52 units/g (O. gratisinum) and 2283.75 (G. max) - 2980.35 units/g (Z. officinale) respectively, when compared to their control 2841.99 and 2253.40 units/g respectively. At $2 \%$ addition the highest value in kulikuli and kokoro 3840.43 units/g (Z. officinale); 2930.35 units/g (sweet basil) respectively. These results are higher than those reports for sun and freeze dried of some edible leafy vegetables [12] but compared well with the result on Amaranthus specie (3129.9 units/g) [12]. Despite the heat treatment during value addition, ginger and sweet basil substantially increased the vitamin A of the under study snacks. Vitamin A is an oxidation product of half the number of carotene found mostly in plant foods with molecular formula $\mathrm{C}_{40} \mathrm{H}_{56}$. It is required for normal growth and for adequate maintenance of well being, the deficiency causes of eye problem known as Xeropthalmia [12].

The values for vit. $\mathrm{C}$ (ascorbic acid) in the control and value-added kulikuli and kokoro are shown in Table 4 . Ascorbic acid content at 1 or $2 \%$ addition in kulikuli and kokoro were not significantly different $(\mathrm{P}>0.05)$. However, result of 1 or $2 \%$ change were significantly $(\mathrm{P}<0.05)$ different with highest percentage change at $2 \%, 67.72 \%$ (H. sabdariffa) and 36.71\% (orange peel) kulikuli and kokoro respectively. Similar results have been reported for some traditional foods [2]. The daily requirement for vitamin C as reported by [13] ranges

Table 3: $\quad$ Vitamin A units/g of the control and value added snacks.

\begin{tabular}{llllll}
\hline \multicolumn{1}{c}{$\begin{array}{c}\text { Source of } \\
\text { nutraceuticals }\end{array}$} & Control & 1\% addition & $\begin{array}{c}\text { Percentage } \\
\text { change at } \% \\
\text { addition }\end{array}$ & 2\% addition & $\begin{array}{c}\text { Percentage } \\
\text { change at } \% \\
\text { addition }\end{array}$ \\
\hline Kulikuli & 2841.99 & $2914.35^{\mathrm{a} \pm} 1.6$ & $2.55^{\mathrm{b}} 1.6$ & $3014.35^{\mathrm{b}} \pm 2.5$ & $6.06^{\mathrm{ab}} \pm 2.5$ \\
Pepper & 2841.99 & $2919.50^{\mathrm{a}} \pm 2.0$ & $2.73^{\mathrm{b}} \pm 2.0$ & $2949.50^{\mathrm{a}} \pm 2.1$ & $3.78^{\mathrm{a}} \pm 2.1$ \\
Cocoa powder & 2841.99 & $2892.10^{\mathrm{a}} \pm 1.7$ & $1.76^{\mathrm{a}} \pm 1.7$ & $2917.10^{\mathrm{a}} \pm 1.9$ & $2.64^{\mathrm{a}} \pm 1.9$ \\
Soya beans & 2841.99 & $2940.20^{\mathrm{a}} \pm 1.4$ & $3.46^{\mathrm{ab}} \pm 1.4$ & $2973.20^{\mathrm{a}} \pm 2.8$ & $4.62^{\mathrm{b}} \pm 2.8$ \\
Orange peel & 2841.99 & $3520.39^{\mathrm{b}} \pm 1.8$ & $20.35^{\mathrm{c}} \pm 1.8$ & $3840.40^{\mathrm{b}} \pm 3.1$ & $35.13^{\mathrm{abc}} \pm 3.1$ \\
Ginger & 2841.99 & $3582.52^{\mathrm{b}} \pm 1.9$ & $26.07^{\mathrm{abc}} \pm 1.9$ & $3712.50^{\mathrm{b}} \pm 1.9$ & $30.63^{\mathrm{c}} \pm 1.9$ \\
Sweet basil & 2841.99 & $2918.98^{\mathrm{a}} \pm 0.9$ & $2.71^{\mathrm{a}} \pm 0.9$ & $2973.10^{\mathrm{a}} \pm 2.9$ & $4.61^{\mathrm{b}} \pm 2.9$ \\
Roselle & & & & & \\
\hline Kokoro & 2253.40 & $2290.35^{\mathrm{a}} \pm 2.6$ & $1.64^{\mathrm{b}} \pm 2.6$ & $2305.30^{\mathrm{a}} \pm 2.0$ & $3.30^{\mathrm{b}} \pm 2.0$ \\
Pepper & 2253.40 & $2295.10^{\mathrm{a}} \pm 2.7$ & $1.83^{\mathrm{b}} \pm 2.7$ & $2325.10^{\mathrm{a}} \pm 2.8$ & $3.18^{\mathrm{b}} \pm 2.8$ \\
Cocoa powder & 2253.40 & $2283.75^{\mathrm{a}} \pm 3.1$ & $1.35^{\mathrm{a}} \pm 3.1$ & $2294.65^{\mathrm{a}} \pm 2.9$ & $1.83^{\mathrm{a}} \pm 2.9$ \\
Soya beans & 2253.40 & $2334.06^{\mathrm{a}} \pm 2.5$ & $3.58^{\mathrm{ab}} \pm 2.5$ & $2359.05^{\mathrm{a}} \pm 2.7$ & $4.68^{\mathrm{ab}} \pm 2.7$ \\
Orange peel & 2253.40 & $2715.40^{\mathrm{b}} \pm 2.7$ & $20.50^{\mathrm{abc}} \pm 2.7$ & $2835.40^{\mathrm{b}} \pm 2.5$ & $25.33^{\mathrm{ab}} \pm 2.5$ \\
Ginger & 2253.40 & $2780.35^{\mathrm{b}} \pm 2.5$ & $23.38^{\mathrm{d}} \pm 2.5$ & $2930.35^{\mathrm{b}} \pm 3.1$ & $30.04^{\mathrm{d}} \pm 3.1$ \\
Sweet basil & 2253.40 & $2404.10^{\mathrm{a}} \pm 2.0$ & $6.69^{\mathrm{c}} \pm 2.0$ & $2444.10^{\mathrm{a}} \pm 2.5$ & $8.46^{\mathrm{c}} \pm 2.5$ \\
Roselle & & & & \\
\hline
\end{tabular}

Means with different superscripts in the same row differs significantly $(\mathrm{P} \leq 0.05)$. 
Table 4: $\quad$ Ascorbic acid mg/g in the control and value added snacks.

\begin{tabular}{llllll}
\hline \multicolumn{1}{c}{$\begin{array}{c}\text { Source of } \\
\text { nutraceuticals }\end{array}$} & Control & $\begin{array}{c}1 \% \\
\text { addition }\end{array}$ & $\begin{array}{c}\text { Percentage } \\
\text { change at } 1 \% \\
\text { addition }\end{array}$ & $2 \%$ addition & $\begin{array}{c}\text { Percentage change } \\
\text { at } 2 \% \text { addition }\end{array}$ \\
\hline $\begin{array}{l}\text { Kulikuli } \\
\text { Pepper }\end{array}$ & 1.58 & $1.60^{\mathrm{a} \pm} 0.9$ & $1.26^{\mathrm{a} \pm} 0.9$ & $1.73^{\mathrm{a} \pm} 0.9$ & $9.49^{ \pm} 0.9$ \\
Cocoa powder & 1.58 & $1.65^{\mathrm{a}} \pm 1.2$ & $4.43^{\mathrm{ab}} \pm 1.2$ & $1.89^{\mathrm{a}} \pm 2.1$ & $19.6 \pm 2.1$ \\
Soya beans & 1.58 & $1.80^{\mathrm{a}} \pm 1.5$ & $13.92^{\mathrm{abc}} \pm 1.5$ & $2.00^{\mathrm{a}} \pm 2.6$ & $26.58 \pm 2.6$ \\
Orange peel & 1.58 & $1.83^{\mathrm{a}} \pm 0.9$ & $15.82^{\mathrm{abc}} \pm 0.9$ & $2.12^{\mathrm{a}} \pm 1.8$ & $34.17 \pm 1.8$ \\
Ginger & 1.58 & $1.72^{\mathrm{b}} \pm 1.0$ & $8.86^{\mathrm{c}} \pm 1.0$ & $1.95^{\mathrm{a}} \pm 1.9$ & $23.41 \pm 1.9$ \\
Sweet basil & 1.58 & $1.96^{\mathrm{b}} \pm 1.2$ & $24.05^{\mathrm{d}} \pm 1.2$ & $2.25^{\mathrm{a}} \pm 2.0$ & $42.41 \pm 2.0$ \\
Roselle & 1.58 & $2.03^{\mathrm{a}} \pm 1.1$ & $28.48^{\mathrm{e}} \pm 1.1$ & $2.65^{\mathrm{b}} \pm 1.8$ & $67.72 \pm 1.8$ \\
\hline Kokoro & & & & & \\
Pepper & 4.44 & $5.39^{\mathrm{b} \pm} 1.5$ & $21.40^{\mathrm{abc} \pm} 1.5$ & $5.75^{\mathrm{b} \pm} 1.5$ & $29.50^{\mathrm{c} \pm} 1.5$ \\
Cocoa powder & 4.44 & $4.52^{\mathrm{a}} \pm 1.6$ & $1.80^{\mathrm{a}} \pm 1.6$ & $4.94^{\mathrm{a}} \pm 2.1$ & $11.26^{\mathrm{b}} \pm 2.1$ \\
Soya beans & 4.44 & $5.32^{\mathrm{b}} \pm 1.2$ & $19.82^{\mathrm{c}} \pm 1.2$ & $6.05^{\mathrm{b}} \pm 2.7$ & $36.26^{\mathrm{d}} \pm 2.7$ \\
Orange peel & 4.44 & $5.29 \pm 0.9$ & $19.14^{\mathrm{c}} \pm 0.9$ & $6.07^{\mathrm{b}} \pm 3.1$ & $36.71^{\mathrm{d}} \pm 3.1$ \\
Ginger & 4.44 & $4.49^{\mathrm{a}} \pm 1.5$ & $1.13^{\mathrm{a}} \pm 1.5$ & $4.53^{\mathrm{a}} \pm 2.0$ & $2.02^{\mathrm{a}} \pm 2.0$ \\
Sweet basil & 4.44 & $5.22^{\mathrm{b}} \pm 1.7$ & $17.56^{\mathrm{ab}} \pm 1.7$ & $5.45^{\mathrm{b}} \pm 2.0$ & $22.74^{\mathrm{abc}} \pm 2.0$ \\
Roselle & 4.44 & $4.63^{\mathrm{a}} \pm 1.1$ & $4.27^{\mathrm{b}} \pm 1.1$ & $4.90^{\mathrm{a}} \pm 1.9$ & $10.36^{\mathrm{b}} \pm 1.9$ \\
\hline
\end{tabular}

Means with different superscripts in the same row differs significantly $(\mathrm{P} \leq 0.05)$.

Table 5: $\quad$ Phenolic content mg/g in the control and value added snacks.

\begin{tabular}{llllll}
\hline \multicolumn{1}{c}{$\begin{array}{c}\text { Source of } \\
\text { nutraceuticals }\end{array}$} & Control & $1 \%$ addition & $\begin{array}{c}\text { Percentage } \\
\text { change at } 1 \% \\
\text { addition }\end{array}$ & 2\% addition & $\begin{array}{c}\text { Percentage } \\
\text { change at } 2 \% \\
\text { addition }\end{array}$ \\
\hline $\begin{array}{l}\text { Kulikuli } \\
\text { Pepper }\end{array}$ & 1.38 & $1.40^{\mathrm{a} \pm} 0.9$ & $1.45^{\mathrm{b} \pm} 0.9$ & $1.45^{\mathrm{a} \pm} 0.4$ & $5.07^{\mathrm{b} \pm} 0.4$ \\
Cocoa powder & 1.38 & $1.39^{\mathrm{a}} \pm 0.5$ & $0.72^{\mathrm{a}} \pm 0.5$ & $1.40^{\mathrm{a}} \pm 0.9$ & $1.44^{\mathrm{a}} \pm 0.9$ \\
Soya beans & 1.38 & $1.39^{\mathrm{a}} \pm 0.6$ & $0.72^{\mathrm{a}} \pm 0.6$ & $1.40^{\mathrm{a}} \pm 1.0$ & $1.44^{\mathrm{a} \pm 1.0}$ \\
Orange peel & 1.38 & $1.40^{\mathrm{a}} \pm 0.3$ & $1.45^{\mathrm{b}} \pm 0.3$ & $1.50^{\mathrm{a}} \pm 0.8$ & $8.70^{\mathrm{c}} \pm 0.8$ \\
Ginger & 1.38 & $1.42^{\mathrm{a}} \pm 0.5$ & $2.90^{\mathrm{ab}} \pm 0.5$ & $1.45^{\mathrm{a}} \pm 0.9$ & $5.07^{\mathrm{ab}} \pm 0.9$ \\
Sweet basil & 1.38 & $1.44^{\mathrm{a}} \pm 0.9$ & $4.35^{\mathrm{c}} \pm 0.9$ & $1.52^{\mathrm{a}} \pm 1.1$ & $10.10^{\mathrm{abc}} \pm 1.1$ \\
Roselle & 1.38 & $1.60^{\mathrm{b}} \pm 0.6$ & $15.94^{\mathrm{d}} \pm 0.6$ & $1.66^{\mathrm{a}} \pm 0.9$ & $19.57^{\mathrm{d}} \pm 0.9$ \\
\hline Kokoro & & & & & \\
Pepper & 10.84 & $15.60^{\mathrm{ab} \pm} 2.0$ & $38.38^{\mathrm{abc} \pm} 2.0$ & $15.70^{\mathrm{b} \pm} 2.5$ & $44.83^{\mathrm{abc} \pm 2.5}$ \\
Cocoa powder & 10.84 & $13.18^{\mathrm{a}} \pm 1.9$ & $21.59^{\mathrm{ab}} \pm 1.9$ & $15.52^{\mathrm{b}} \pm 1.5$ & $43.17^{\mathrm{c}} \pm 1.5$ \\
Soya beans & 10.84 & $14.31^{\mathrm{b}} \pm 1.2$ & $32.01^{\mathrm{c}} \pm 1.2$ & $15.50^{\mathrm{b}} \pm 1.7$ & $42.98^{\mathrm{c}} \pm 1.7$ \\
Orange peel & 10.84 & $13.18^{\mathrm{a}} \pm 0.9$ & $21.59^{\mathrm{a}} \pm 0.9$ & $14.50^{\mathrm{a}} \pm 2.1$ & $33.76^{\mathrm{b}} \pm 2.1$ \\
Ginger & 10.84 & $12.70^{\mathrm{a}} \pm 1.2$ & $17.16^{\mathrm{b}} \pm 1.2$ & $13.50^{\mathrm{a}} \pm 1.8$ & $24.53^{\mathrm{a}} \pm 1.8$ \\
Sweet basil & 10.84 & $12.23^{\mathrm{a}} \pm 1.4$ & $12.82^{\mathrm{a}} \pm 1.4$ & $14.53^{\mathrm{a}} \pm 2.0$ & $34.04^{\mathrm{ab}} \pm 2.0$ \\
Roselle & 10.84 & $16.50^{\mathrm{ab}} \pm 1.1$ & $52.21^{\mathrm{d}} \pm 1.1$ & $17.92^{\mathrm{b}} \pm 2.0$ & $65.31^{\mathrm{d}} \pm 2.0$ \\
\hline
\end{tabular}

Means with different superscripts in the same row differs significantly $(\mathrm{P} \leq 0.05)$.

between 45 to $60 \mathrm{mg}$. Value addition increases the vitamin C contents but lower than the daily requirement. This low value after value addition may be attributed to instability of vitamin $C$. However, the amount of vitamin $C$ in the value added snacks compare favourably with those reported for some citrus plant foods [1]. 
The phenolic content mg/g of the control and value-added kulikuli and kokoro are shown in Table 5. Phenolic phytochemicals inhibit autooxidation of unsaturated lipids, thus preventing the formation of oxidized low-density lipoprotein (LDL) which is considered to induce cardiovascular disease [2]. Phenolic content of the value added snacks at 1 or $2 \%$ addition in kulikuli were not significantly different $(\mathrm{P}>0.05)$ while kokoro were significantly different $(\mathrm{P}$ $<0.05$ ). Among all the nutraceuticals added to kulikuli and kokoro H. sabdariffa had the highest value $\mathrm{mg} / \mathrm{g}$ of phenolic content at $1 \%(1.60 ; 16.50 \mathrm{mg} / \mathrm{g})$ and at $2 \%(1.65 ; 17.92 \mathrm{mg} / \mathrm{g})$ respectively. The least increased $1.39 \mathrm{mg} / \mathrm{g}$ was found at $1 \%$ addition of cocoa powder and soyabeans to kulikuli. These values were generally higher than those reported by [2] on some tropical green leafy vegetables.

The result of the free radical scavenging ability Table 6 of all the value added kulikuli and kokoro at 1 or $2 \%$ addition ranged from $1.26 \mathrm{mg} / \mathrm{g}$ (sweet basil) in kokoro to $4.00 \mathrm{mg} / \mathrm{g}$ (H. sabdariffa) in kulikuli compare to the control 3.5mg/g to $0.96 \mathrm{mg} / \mathrm{g}$ respectively. These values are within the range with the report [4] for some tropical green leafy vegetables. Value addition caused significant increase $(\mathrm{P}<0.05)$ at 1 or $\%$ change in the free radical scavenging ability of all the value added snacks.

Table 6: $\quad$ Free radical scavenging ability (\%) of the control and value added snacks.

\begin{tabular}{llllll}
\hline $\begin{array}{c}\text { Source of } \\
\text { nutraceuticals }\end{array}$ & Control & $1 \%$ addition & $\begin{array}{c}\text { Percentage } \\
\text { change at } 1 \% \\
\text { addition }\end{array}$ & $\begin{array}{c}2 \% \\
\text { addition }\end{array}$ & $\begin{array}{c}\text { Percentage } \\
\text { change at 2\% } \\
\text { addition }\end{array}$ \\
\hline Kulikuli & & & & & \\
Pepper & 3.51 & $3.61^{\mathrm{a}} 0.9$ & $2.85^{\mathrm{a}} 0.9$ & $3.67^{\mathrm{a} \pm} 2.5$ & $4.55^{ \pm} 2.5$ \\
Cocoa powder & 3.51 & $3.85^{\mathrm{a}} \pm 1.6$ & $9.69^{\mathrm{ab}} \pm 1.6$ & $3.89^{\mathrm{a}} \pm 2.1$ & $10.83 \pm 2.1$ \\
Soya beans & 3.51 & $3.70^{\mathrm{a}} \pm 1.8$ & $5.41^{\mathrm{b}} \pm 1.8$ & $3.80^{\mathrm{a}} \pm 2.0$ & $8.26 \pm 2.0$ \\
Orange peel & 3.51 & $3.58^{\mathrm{a}} \pm 1.9$ & $1.99^{\mathrm{a}} \pm 1.9$ & $3.60^{\mathrm{a}} \pm 1.8$ & $2.56^{\mathrm{a}} \pm 1.8$ \\
Ginger & 3.51 & $3.90^{\mathrm{a}} \pm 1.8$ & $11.11 \pm 1.8$ & $3.95 \pm 1.7$ & $12.54 \pm 1.7$ \\
Sweet basil & 3.51 & $3.56^{\mathrm{a}} \pm 1.3$ & $1.42^{\mathrm{a}} \pm 1.3$ & $3.60^{\mathrm{a}} \pm 2.2$ & $2.56^{\mathrm{a}} \pm 2.2$ \\
Roselle & 3.51 & $3.95^{\mathrm{a}} \pm 1.4$ & $12.54 \pm 1.4$ & $4.00 \pm 2.0$ & $13.96 \pm 2.0$ \\
\hline Kokoro & & & & & \\
Pepper & 0.96 & $1.62^{\mathrm{a}} \pm 1.2$ & $68.75^{\mathrm{ab}} \pm 1.7$ & $1.74^{\mathrm{a}} \pm 1.2$ & $81.13^{\mathrm{c}} \pm 1.8$ \\
Cocoa powder & 0.96 & $1.35^{\mathrm{a}} \pm 1.8$ & $40.63^{\mathrm{b}} \pm 1.2$ & $1.59^{\mathrm{a}} \pm 2.1$ & $65.63^{\mathrm{b}} \pm 0.9$ \\
Soya beans & 0.96 & $1.42^{\mathrm{a}} \pm 2.0$ & $47.92^{\mathrm{b}} \pm 1.3$ & $1.56^{\mathrm{a}} \pm 1.6$ & $62.50^{\mathrm{b}} \pm 1.5$ \\
Orange peel & 0.96 & $1.60^{\mathrm{a}} \pm 1.5$ & $66.66^{\mathrm{ab}} \pm 1.9$ & $1.68^{\mathrm{a}} \pm 1.8$ & $75.00^{\mathrm{ab}} \pm 1.4$ \\
Ginger & 0.96 & $1.67^{\mathrm{a}} \pm 1.8$ & $73.96^{\mathrm{c}} \pm 2.0$ & $1.72^{\mathrm{a}} \pm 1.5$ & $79.16^{\mathrm{c}} \pm 1.4$ \\
Sweet basil & 0.96 & $1.26^{\mathrm{a}} \pm 2.1$ & $31.25^{\mathrm{a}} \pm 1.5$ & $1.40^{\mathrm{a}} \pm 1.6$ & $45.83^{\mathrm{a}} \pm 1.6$ \\
Roselle & 0.96 & $1.69^{\mathrm{a}} \pm 1.8$ & $76.04^{\mathrm{c}} \pm 1.4$ & $1.74^{\mathrm{a}} \pm .1 .3$ & $81.25^{\mathrm{c}} \pm 1.1$ \\
\hline
\end{tabular}

Means with different superscripts in the same row differs significantly $(\mathrm{P} \leq 0.05)$.

\section{Conclusion}

The study clearly shows the high potential of enriched traditional snacks in the provision of adequate level of acceptance, vitamins and interesting antioxidant properties. Considering their easy accessibility and relatively cheap, consumption of snacks and cakes fortified with these nutraceuticals could be a 
strategic approach towards management of micro-nutrient deficiency and good health.

\section{Acknowledgement}

We are grateful to the Centre of Excellence in Food Security (World Bank sponsored project), Federal University of Technology, Akure, Nigeria for providing the financial support to this research work.

\section{References}

[1] Zhang, Z.; Chnag, O.; Zhu, M.; Huang, Y.; Ho, W.K.K. and Chen, Z. Characterisation of antioxidants present in hawthorn fruits. J. Nutr. Biochem. (12) 144-152 (2001).

[2] Oboh, G. Effect of blanching on the antioxidant property of some tropical green leafy vegetables. Lebensm-Wiss. U. - Technol 38 (5): 513-517 (2005a).

[3] Potter, N.N. Hedonic scale: Food Science. The AVI Publishing Company Inc., Wespot, C.T. (1968).

[4] Wheeler, V.E. and Ferrel, R.E. A method for phytic acid determination in wheal fraction. Cereal Chem., 48, 312-16 (1971).

[5] Makkar, H.P.S.; Blummel, M.; Bowwy, N.K. and Becke, K. Determination of tannins and correlation with chemical and protein precipitation method. J. Sci. Food Agric. 61, 161-185 (1993).

[6] Association of Official Analytical Chemistry. Official Methods of Analysis International. $17^{\text {th }}$ edn. AOAC International, Maryland, U.S.A. (2000).

[7] Singleton, V.L.; Orthofer, R. and Lamuela-Raventos, R.M. Analysis of total phenols and other oxidation substrates and antioxidants by means of Folin-Ciocaltau reagent. Methods in Enzymology, (299), 152-179. (1999).

[8] Ursini, F.; Maiorino, M.; Morrazoni, P.; Roverl, A. and Pifferi, G. A novel antioxidant (1dB 1031) affecting molecular mechanisms of cellular. Free Radical Biology and Medicine. 547-553 (1994).

[9] Zar, J.H. Biostatistical analysis. Prentice-Hall: USA. (1984).

[10] Oboh, G. and Akindahunsi, A.A. Biochemical changes in cassava products (Flour and Gari) subjected to Saccharomyces cerevisae solid Media Fermentation. Food Chemistry 82 (4), 599-602, (2003).

[11] Aletor, V.A. Compositional studies on edible tropical species of mushroom. J. Food Chemistry 54: 265-268, (1995).

[12] Abiodun, A.B. Effect of drying on the nutrient and antinutrient constituents of some edible leafy vegetables. M. Tech. Thesis, Department of Chemistry, The Federal University of Technology, Akure, Nigeria. pp. 8085, (2012).

[13] WHO, Management of severe malnutrition. A manual for physicians and other senior health workers. World Health Organisation, Geneva, 60p (1999). 